\title{
ROBUST STABILIZATION USING A SAMPLED-DATA STRATEGY OF UNCERTAIN NEUTRAL STATE-DELAYED SYSTEMS SUBJECT TO INPUT LIMITATIONS
}

\author{
NABil EL FEZAZI ${ }^{a}$, FATIMA EL HAOUSSI $^{a, b}$, El Houssaine TISSIR ${ }^{a}$, Teresa ALVAREZ $^{c}$, \\ FERNANDO TADEO ${ }^{c, *}$
}

\author{
${ }^{a}$ Faculty of Sciences Dhar El Mehraz \\ Sidi Mohammed Ben Abdellah University, LESSI, BP 1796, Fes-Atlas, Morocco \\ e-mail: fizazi.99@gmail.com, elhoussaine.tissir@usmba.ac.ma \\ ${ }^{b}$ Multidisciplinary Faculty \\ Mohammed the First University, BP 300, Selouane 62700, Nador, Morocco \\ e-mail: elhaous_fati@yahoo.fr \\ ${ }^{c}$ Industrial Engineering School \\ University of Valladolid, Dr. Mergelina S/N, 47005 Valladolid, Spain \\ e-mail: tere@autom.uva.es, fernando.tadeo@uva.es
}

\begin{abstract}
Stabilization of neutral systems with state delay is considered in the presence of uncertainty and input limitations in magnitude. The proposed solution is based on simultaneously characterizing a set of stabilizing controllers and the associated admissible initial conditions through the use of a free weighting matrix approach. From this mathematical characterization, state feedback gains that ensure a large set of admissible initial conditions are calculated by solving an optimization problem with LMI constraints. Some examples are presented to compare the results with previous approaches in the literature.
\end{abstract}

Keywords: robust stabilization, sampled-data, time-delay systems, input saturation, admissible initial conditions, LMI.

\section{Introduction}

Time-delay systems have theoretical and practical importance because delays appear in epidemic, population dynamics and economy models, and in many other practical areas. In fact, delays generally lead to poor closed-loop system performance and instabilities. Therefore, closed-loop stability analysis of time-delay systems is being extensively studied (see, e.g., the works of Gu et al. (2003), Thuan et al. (2012), Tissir (2010), Zakaria et al. (2015) or Zhang et al. (2005) and the references therein). Moreover, practical control systems are subject to input limitations, because of the existence of technological and safety constraints (see ElFezazi et al., 2016; 2015; El Haoussi and Tissir, 2010; Liu, 2005; Mesquine, 2004). Input limitations are known to be a source of performance degradation, generated limit

\footnotetext{
*Corresponding author
}

cycles, multiple equilibrium points and even instability. Therefore they should be taken into account during the design (see, e.g., the works of Tissir and Hmamed (1992), Cao et al. (2002), Tarbouriech et al. (2003) or El Fezazi et al. (2017) and the references therein).

The design of controllers in simultaneous presence of time-delays and input limitations makes closed-loop stabilization a difficult problem. Nonetheless, some works addressing stabilization in the presence of input saturation can be found in the literature. For example, Tissir and Hmamed (1992) proposed some conditions for stabilization under state feedback. However, the set of admissible initial conditions for which asymptotic stability is ensured in the presence of input saturation (called the domain of attraction) was not considered. In the work of Tarbouriech et al. (2003), the synthesis of stabilizing static anti-windup loops was addressed for the case of fixed delays. Cao et al. (2002) proposed a method 
for computing stabilizing state feedback control laws aimed at enlarging estimates of the domain of attraction, based on the use of polytopic differential inclusions to describe the behavior of the closed-loop system with saturating inputs. Based on this ideas, in a recent work (El Fezazi et al., 2017) we proposed, for neutral systems, to use a generalized sector approach to modelling the saturation effects, and a method for computing stabilizing state feedback controls in the continuous time domain: that proposal is completed here for sampled-data control, which is more relevant for practical problems. Sampled-data control in the presence of input saturations has been studied, e.g., by Fridman et al. (2004) or Seuret and Da Silva (2012). However, to the best of our knowledge, there is a lack of works dealing with the problem of sampled-data control in the presence of input saturations for the class of systems studied in this paper (Wang et al., 2014; 2015). Moreover, it has not been yet sufficiently taken into account that, for practical problems, it is important to consider uncertainty in the models, as they are always an approximation of the reality, so uncertainty will be explicitly considered here.

Thus, in this paper, the robust stabilization problem for neutral systems with time-varying delay and input magnitude limitations is solved when the controller is based on sampling. By incorporating Lyapunov-Krasovskii (L-K) functional theory, the free-weighting matrix technique, integral inequalities and a function to describe decentralized dead-zone nonlinearities, efficient stabilization conditions are obtained in terms of LMIs for a general class of uncertainties. An optimization problem is formulated with the aim of computing stabilizing state feedback control laws: this optimization looks for the maximal delay bound for which a stabilizing control law can be found. On the other hand, when the open-loop system is unstable, the optimization objective consists in finding a control law that maximizes an estimate of the domain of attraction, or, alternatively, ensures the stability for a given set of admissible initial states.

The rest of this paper is organized as follows. Section 2 formulates the stabilization of uncertain neutral time-delay systems with input limitations. The main results are presented in Section 3. Practical optimization problems are formulated in Section 4. Finally, some numerical examples are solved to illustrate the results developed in this paper.

The following notation will be used: the Banach space of continuous vector functions mapping the interval $\left[-h_{m}, 0\right]$ into $\mathbb{R}^{n}$ is denoted by $\mathcal{C}_{h_{m}}=\mathcal{C}\left(\left[-h_{m}, 0\right], \mathbb{R}^{n}\right)$, $K_{i}$ denotes the $i$-th row of $K, \bar{\lambda}(P)$ is the maximal eigenvalue of matrix $P$.

\section{Problem formulation and preliminaries}

Consider the following uncertain neutral time-delay system:

$$
\begin{aligned}
\dot{x}(t)-C \dot{x}(t-\tau(t))= & \widehat{A} x(t)+\widehat{A}_{d} x(t-\tau(t)) \\
& +\widehat{B} u(t)+B_{w} w(t), \\
z(t)= & C_{z} x(t), \\
x(t)= & \phi(t), \quad \phi(t) \in \mathcal{C}_{h_{m}},
\end{aligned}
$$

where $x(t) \in \mathbb{R}^{n}, u(t) \in \mathbb{R}^{m}, w(t) \in \mathbb{R}^{r}$ and $z(t) \in \mathbb{R}^{s}$ are, respectively, the state, the control, the disturbance and the output vectors. Here $w(t)$ is assumed to be bounded with finite energy, that is, $w(t) \in \mathcal{L}_{2}$. Hence, for a scalar $\omega$, the disturbance $w(t)$ is bounded as follows:

$$
\|w(t)\|_{2}^{2}=\int_{0}^{\infty} w^{T}(t) w(t) \mathrm{d} t \leq \omega^{-1}<\infty .
$$

The delay $\tau(t)$ is a positive integer that can be time dependent, but satisfying $0 \leq \tau(t) \leq h_{m}$ and $0 \leq \dot{\tau}(t) \leq$ $d<1$, where $h_{m}$ and $d$ are known positive and finite integers.

The uncertain matrices $\widehat{A}, \widehat{A}_{d}$ and $\widehat{B}$ are decomposed as follows: $\widehat{A}=A+\Delta A(t), \widehat{A}_{d}=A_{d}+\Delta A_{d}(t)$ and $\widehat{B}=B+\Delta B(t)$, where $\Delta A(t), \Delta A_{d}(t)$ and $\Delta B(t)$ are system uncertainties, structured as

$$
\left[\Delta A(t) \quad \Delta A_{d}(t) \quad \Delta B(t)\right]=D F(t)\left[\begin{array}{lll}
E_{0} & E_{1} & E_{2}
\end{array}\right],
$$

where $D, E_{0}, E_{1}$ and $E_{2}$ are known real matrices of appropriate dimensions, and $F(t)$ denotes time-varying uncertainties with Lebesgue-measurable elements that fulfill

$$
F^{T}(t) F(t) \leq I, \quad \forall t \geq 0 .
$$

Moreover, $C, A, A_{d}, B, B_{w}$ and $C_{z}$ are constant real matrices of appropriate dimensions.

Special focus in this paper is on the fact that the controller uses sampled signals: the state is then measured by a sampler at discrete-time instants $t_{k=0,1, \ldots}$, satisfying

$$
0=t_{0}<\cdots<t_{k}<\cdots,
$$

with $t_{k+1}-t_{k} \leq h_{k}$, where $h_{k}$ indicates the maximal sampling period at instant $k$.

We assume that outputs and control signals are measured and transmitted quickly, compared with the system delay and time constants, and the communication delay is small enough to be ignored (the communication channel is ideal); then, the control law is expressed by $u(t)=u\left(t_{k}\right), t_{k} \leq t<t_{k+1}$. In order to mathematically represent input sampling, the sampling instants $t_{k}$ are represented by a time-delay $d_{k}(t)=t-t_{k}$, which satisfies $0 \leq d_{k}(t) \leq h_{k}$, and $\dot{d}_{k}(t)=1$. 
Moreover, each component of the input vector $u\left(t_{k}\right)$ is subject to magnitude limitations defined by $\left|u_{i}\left(t_{k}\right)\right| \leq$ $u_{0_{i}}, u_{0_{i}}>0$, where $i=1, \ldots, m$. Furthermore, to generate the desired time responses systematically, we adopt a standard control problem for the system (1): due to the control bounds, the effective control signal to be applied to the system (1) is given by

$$
u\left(t_{k}\right)=\operatorname{sat}\left(K x\left(t_{k}\right)\right),
$$

where the saturation function $\operatorname{sat}\left(K x\left(t_{k}\right)\right)$ is

$$
\left[\operatorname{sat}\left(K_{1} x\left(t_{k}\right)\right) \ldots \operatorname{sat}\left(K_{m} x\left(t_{k}\right)\right)\right]^{T}
$$

with

$$
\operatorname{sat}\left(K_{i} x\left(t_{k}\right)\right)=\operatorname{sign}\left(K_{i} x\left(t_{k}\right)\right) \min \left\{u_{0_{i}},\left|K_{i} x\left(t_{k}\right)\right|\right\} .
$$

Hence, the closed-loop system (1) is

$$
\begin{aligned}
\dot{x}(t)-C \dot{x}(t-\tau(t))= & \widehat{A} x(t)+\widehat{A}_{d} x(t-\tau(t)) \\
& +\widehat{B} \operatorname{sat}\left(K x\left(t-d_{k}(t)\right)\right) \\
& +B_{w} w(t), \\
z(t)= & C_{z} x(t) .
\end{aligned}
$$

The nominal system, ignoring the unknown parameters in (5), is given by

$$
\begin{aligned}
\dot{x}(t)-C \dot{x}(t-\tau(t))= & A x(t)+A_{d} x(t-\tau(t)) \\
& +B \operatorname{sat}\left(K x\left(t-d_{k}(t)\right)\right) \\
& +B_{w} w(t), \\
z(t)= & C_{z} x(t) .
\end{aligned}
$$

To mathematically treat the control limitations, we define $\psi\left(K x\left(t-d_{k}(t)\right)\right)=K x\left(t-d_{k}(t)\right)-\operatorname{sat}(K x(t-$ $\left.\left.d_{k}(t)\right)\right)$, which corresponds to a decentralized dead-zone nonlinearity. Thus, the closed-loop system (6) can be written as

$$
\begin{aligned}
\dot{x}(t)-C \dot{x}(t-\tau(t))= & A x(t)+A_{d} x(t-\tau(t)) \\
& +B K x\left(t-d_{k}(t)\right)+B_{w} w(t) \\
& -B \psi\left(K x\left(t-d_{k}(t)\right)\right), \\
z(t)= & C_{z} x(t) .
\end{aligned}
$$

The following auxiliary function will also be used:

$$
J(t)=\dot{V}(t)+\frac{1}{\gamma} z^{T}(t) z(t)-w^{T}(t) w(t) .
$$

We now derive a controller (4) such that the system (7) is asymptotically stable and satisfies the following condition for the performance index:

$$
\int_{0}^{\infty}\left[\frac{1}{\gamma} z^{T}(t) z(t)-w^{T}(t) w(t)\right] \mathrm{d} t<0,
$$

where the prescribed scalar $\gamma$ should be as small as possible.

The $\mathrm{L}-\mathrm{K}$ functional candidate is selected to be

$$
\begin{aligned}
V(t)= & x^{T}(t) P x(t)+\int_{t-\tau(t)}^{t} x^{T}(s) Q_{1} x(s) \mathrm{d} s \\
& +\int_{t-d_{k}(t)}^{t} x^{T}(s) Q_{2} x(s) \mathrm{d} s \\
& +\int_{-\tau(t)}^{0} \int_{t+\theta}^{t} \dot{x}^{T}(s) R_{1} \dot{x}(s) \mathrm{d} s \mathrm{~d} \theta \\
& +\int_{-d_{k}(t)}^{0} \int_{t+\theta}^{t} \dot{x}^{T}(s) R_{2} \dot{x}(s) \mathrm{d} s \mathrm{~d} \theta \\
& +\int_{t-\tau(t)}^{t} \dot{x}^{T}(s) W \dot{x}(s) \mathrm{d} s
\end{aligned}
$$

The following lemmas are going to be used.

Lemma 1. (Peterson, 1987) Let $\Omega, \Upsilon$, and $\Phi$ be real matrices of appropriate dimensions. Then, for $F(t)$ satisfying (3),

$$
\Omega+\Upsilon F(t) \Phi+\Phi^{T} F^{T}(t) \Upsilon^{T}<0
$$

if and only if there exists some $\varepsilon>0$ such that

$$
\Omega+\varepsilon \Upsilon \Upsilon^{T}+\varepsilon^{-1} \Phi^{T} \Phi<0 .
$$

Lemma 2. (Zhang et al., 2005) For any free weighting matrices $Y_{1}$ and $Y_{2}$ of appropriate dimensions, the following bounding inequality holds:

$$
\begin{aligned}
& -\int_{t-\tau(t)}^{t} \dot{x}^{T}(s) R_{1} \dot{x}(s) \mathrm{d} s \\
& \quad \leq \xi^{T}(t)\left(\mathbb{Y}^{T}+\mathbb{I} \mathbb{Y}^{T}+\tau(t) \mathbb{Y} R_{1}^{-1} \mathbb{Y}^{T}\right) \xi(t),
\end{aligned}
$$

where

$$
\begin{gathered}
\xi(t)=\left[\begin{array}{c}
x(t) \\
x(t-\tau(t))
\end{array}\right], \\
\mathbb{Y}=\left[\begin{array}{c}
Y_{1} \\
Y_{2}
\end{array}\right], \quad \mathbb{I}=\left[\begin{array}{c}
I \\
-I
\end{array}\right] .
\end{gathered}
$$

Lemma 3. (Tarbouriech et al., 2003) Given a matrix $G$, define the following polyhedral set:

$$
\begin{aligned}
\mathcal{S}=\left\{x(t) \in \mathbb{R}^{n}:\left|\left(K_{i}-G_{i}\right) x(t)\right|\right. & \leq u_{0_{i}} \\
i & =1, \ldots, m,\} .
\end{aligned}
$$

If $x(t) \in \mathcal{S}$, the relation

$$
\psi^{T}(K x(t)) T_{0}[\psi(K x(t))-G x(t)] \leq 0
$$

is satisfied for any diagonal positive matrix $T_{0}$. 
An ellipsoid $D_{e}$ is characterized as follows:

$$
D_{e}=\left\{x(t) \in \mathbb{R}^{n}: x^{T}(t) P x(t) \leq \beta^{-1}\right\} .
$$

where $\beta$ is a positive scalar and $P>0$. An estimate of the domain of attraction $\Xi \subset \Phi$ is

$$
\Xi=\left\{\phi_{i}(t) \in \mathcal{C}_{h_{m}}: \max \left\|\phi_{i}(t)\right\| \leq \delta\right\},
$$

with the initial condition $x_{0}=\phi_{i}(t),-h_{m} \leq t \leq 0$, and the domain of attraction to the origin is

$$
\Phi=\left\{\phi_{i}(t) \in \mathcal{C}_{h_{m}}: \lim _{t \rightarrow \infty} \phi_{i}\left(t, x_{0}\right)=0\right\}
$$

Problem 1. Based on the analysis and definitions above, the problem solved in this paper is the following: For the system (1), provide a method for designing a controller (4) such that the closed-loop system is robustly stable, and for a given performance index $\gamma>0$, the $H_{\infty}$ norm of the closed-loop transfer function $G(s)$ satisfies

$$
\|G(s)\|_{\infty}^{2}=\frac{\|z(t)\|_{2}^{2}}{\|w(t)\|_{2}^{2}}=\frac{\int_{0}^{\infty} z^{T}(t) z(t) \mathrm{d} t}{\int_{0}^{\infty} w^{T}(t) w(t) \mathrm{d} t}<\gamma
$$

or, equivalently, that the performance index meets $(8)$.

\section{Main results}

Some results are now derived to ensure robust stabilization of the neutral system with input limitations.

3.1. Asymptotic stability. First, some results are derived to ensure asymptotic stabilization of the nominal system for initial conditions within an estimated domain of attraction.

Theorem 1. Assume that there exist symmetric positive definite matrices $\bar{P}, \bar{Q}_{1}, \bar{Q}_{2}, \bar{R}_{1}, \bar{R}_{2}, \bar{W}$, appropriately sized matrices $X, \bar{Y}_{1}, \bar{Y}_{2}, \bar{Z}_{1}, \bar{Z}_{2}, M, U$, a diagonal matrix $S$ of appropriate dimension and a real scalar $\alpha$ satisfying the conditions

$\Omega=\left[\begin{array}{cccccc}\Omega_{11} & * & * & * & * & * \\ \Omega_{21} & \Omega_{22} & * & * & * & * \\ \Omega_{31} & \Omega_{32} & \Omega_{33} & * & * & * \\ \Omega_{41} & \Omega_{42} & 0 & \Omega_{44} & * & * \\ \Omega_{51} & \Omega_{52} & 0 & 0 & \Omega_{55} & * \\ \Omega_{61} & \Omega_{62} & 0 & \Omega_{64} & 0 & \Omega_{66} \\ \Omega_{71} & \Omega_{72} & 0 & 0 & 0 & 0 \\ \Omega_{81} & 0 & \Omega_{83} & 0 & 0 & 0 \\ \Omega_{91} & 0 & 0 & \Omega_{94} & 0 & 0 \\ \Omega_{101} & 0 & 0 & 0 & 0 & 0\end{array}\right.$

$$
\left.\begin{array}{cccc}
* & * & * & * \\
* & * & * & * \\
* & * & * & * \\
* & * & * & * \\
* & * & * & * \\
* & * & * & * \\
\Omega_{77} & * & * & * \\
0 & \Omega_{88} & * & * \\
0 & 0 & \Omega_{99} & * \\
0 & 0 & 0 & \Omega_{1010}
\end{array}\right]<0
$$

$$
\left[\begin{array}{cc}
\bar{P} & * \\
U_{i}-M_{i} & \beta u_{0_{i}}^{2}
\end{array}\right] \geq 0
$$

$$
\beta-\omega \leq 0
$$

$$
\begin{gathered}
\delta^{2}\left(\bar{\lambda}\left(X^{-1} \bar{P} X^{-T}\right)+h_{m} \bar{\lambda}\left(X^{-1} \bar{Q}_{1} X^{-T}\right)\right. \\
+h_{k} \bar{\lambda}\left(X^{-1} \bar{Q}_{2} X^{-T}\right)+\frac{h_{m}^{2}}{2} \bar{\lambda}\left(X^{-1} \bar{R}_{1} X^{-T}\right) \\
\left.+\frac{h_{k}^{2}}{2} \bar{\lambda}\left(X^{-1} \bar{R}_{2} X^{-T}\right)+h_{m} \bar{\lambda}\left(X^{-1} \bar{W} X^{-T}\right)\right] \\
\leq \beta^{-1}-\omega^{-1}
\end{gathered}
$$

where $\delta=\max \|\phi(\theta)\|$ and

$$
\begin{aligned}
\Omega_{11}= & A X^{T}+X A^{T}+\bar{Y}_{1}+\bar{Y}_{1}^{T} \\
& +\bar{Z}_{1}+\bar{Z}_{1}^{T}+\bar{Q}_{1}+\bar{Q}_{2} \\
\Omega_{21}= & -X+\alpha A X^{T}+\bar{P}, \\
\Omega_{31}= & X A_{d}^{T}-\bar{Y}_{1}^{T}+\bar{Y}_{2}, \\
\Omega_{22}= & -\alpha X-\alpha X^{T} \\
& +h_{m} \bar{R}_{1}+h_{k} \bar{R}_{2}+\bar{W}, \\
\Omega_{32}= & \alpha X A_{d}^{T}, \\
\Omega_{33}= & -\bar{Y}_{2}-\bar{Y}_{2}^{T}-(1-d) \bar{Q}_{1} \\
\Omega_{41}= & U^{T} B^{T}-\bar{Z}_{1}^{T}+\bar{Z}_{2}, \quad \Omega_{42}=\alpha U^{T} B^{T}, \\
\Omega_{44}= & -\bar{Z}_{2}-\bar{Z}_{2}^{T}, \\
\Omega_{51}= & X C^{T}, \quad \Omega_{52}=\alpha X C^{T}, \\
\Omega_{55}= & -(1-d) \bar{W}, \quad \Omega_{61}=-S B^{T}, \\
\Omega_{62}= & -\alpha S B^{T}, \quad \Omega_{64}=M, \\
\Omega_{66}= & -2 S^{T}, \quad \Omega_{71}=B_{w}^{T}, \\
\Omega_{72}= & \alpha B_{w}^{T}, \quad \Omega_{77}=-I, \\
\Omega_{81}= & h_{m} \bar{Y}_{1}^{T}, \quad \Omega_{83}=h_{m} \bar{Y}_{2}^{T} \\
\Omega_{88}= & -h_{m} \bar{R}_{1}, \quad \Omega_{91}=h_{k} \bar{Z}_{1}^{T}, \\
\Omega_{94}= & h_{k} \bar{Z}_{2}^{T}, \quad \Omega_{99}=-h_{k} \bar{R}_{2}, \\
\Omega_{101}= & C_{z} X^{T}, \quad \Omega_{1010}=-\gamma I . \\
& =\bar{W}_{1},
\end{aligned}
$$


Then the state feedback control law $K=U X^{-T}$ ensures that

(a) the closed-loop system (6) is asymptotically stable,

(b) the performance index satisfies (10).

Proof. See Appendix A.

In the following, a new result for robust stabilization is provided for the class of systems under study. Thus, based on Theorem 11, some results are now derived to ensure stabilization subject to the uncertainty (2).

\subsection{Robust stability.}

Theorem 2. Assume that there exist symmetric positive definite matrices $\bar{P}, \bar{Q}_{1}, \bar{Q}_{2}, \bar{R}_{1}, \bar{R}_{2}, \bar{W}$, appropriately sized matrices $X, \bar{Y}_{1}, \bar{Y}_{2}, \bar{Z}_{1}, \bar{Z}_{2}, M, U$, a diagonal matrix $S$ of appropriate dimension, a real scalar $\alpha$ and $a$ positive scalar $\varepsilon$ satisfying the conditions

$$
\begin{aligned}
& {\left[\begin{array}{cccccc}
\Omega_{11}^{\prime} & * & * & * & * & * \\
\Omega_{21}^{\prime} & \Omega_{22}^{\prime} & * & * & * & * \\
\Omega_{31} & \Omega_{32} & \Omega_{33} & * & * & * \\
\Omega_{41} & \Omega_{42} & 0 & \Omega_{44} & * & * \\
\Omega_{51} & \Omega_{52} & 0 & 0 & \Omega_{55} & * \\
\Omega_{61} & \Omega_{62} & 0 & \Omega_{64} & 0 & \Omega_{66} \\
\Omega_{71} & \Omega_{72} & 0 & 0 & 0 & 0 \\
\Omega_{81} & 0 & \Omega_{83} & 0 & 0 & 0 \\
\Omega_{91} & 0 & 0 & \Omega_{94} & 0 & 0 \\
\Omega_{101} & 0 & 0 & 0 & 0 & 0 \\
E_{0} X^{T} & 0 & E_{1} X^{T} & E_{2} U & 0 & -E_{2} S^{T}
\end{array}\right.} \\
& \left.\begin{array}{ccccc}
* & * & * & * & * \\
* & * & * & * & * \\
* & * & * & * & * \\
* & * & * & * & * \\
* & * & * & * & * \\
* & * & * & * & * \\
\Omega_{77} & * & * & * & * \\
0 & \Omega_{88} & * & * & * \\
0 & 0 & \Omega_{99} & * & * \\
0 & 0 & 0 & \Omega_{1010} & * \\
0 & 0 & 0 & 0 & -\varepsilon I
\end{array}\right]<0 \\
& {\left[\begin{array}{cc}
\bar{P} & * \\
U_{i}-M_{i} & \beta u_{0_{i}}^{2}
\end{array}\right] \geq 0, \quad \beta-\omega \leq 0} \\
& \delta^{2}\left(\bar{\lambda}\left(X^{-1} \bar{P} X^{-T}\right)+h_{m} \bar{\lambda}\left(X^{-1} \bar{Q}_{1} X^{-T}\right)\right. \\
& +h_{k} \bar{\lambda}\left(X^{-1} \bar{Q}_{2} X^{-T}\right)+\frac{h_{m}^{2}}{2} \bar{\lambda}\left(X^{-1} \bar{R}_{1} X^{-T}\right) \\
& \left.+\frac{h_{k}^{2}}{2} \bar{\lambda}\left(X^{-1} \bar{R}_{2} X^{-T}\right)+h_{m} \bar{\lambda}\left(X^{-1} \bar{W} X^{-T}\right)\right] \\
& \leq \beta^{-1}-\omega^{-1}
\end{aligned}
$$

where $\Omega_{11}^{\prime}=\Omega_{11}+\varepsilon D D^{T}, \Omega_{21}^{\prime}=\Omega_{21}+\varepsilon \alpha D D^{T}$ and $\Omega_{22}^{\prime}=\Omega_{22}+\varepsilon \alpha^{2} D D^{T}$. Then a state feedback control law $K=U X^{-T}$ exists, such that the closed-loop system (5) satisfies

(a) robust stability,

(b) the performance index fulfills (10).

Proof. See Appendix B.

Remark 1. In deriving Theorem 1, slack variables $T_{1}$, $T_{2}, Y_{1}, Y_{2}, Z_{1}, Z_{2}$ are introduced in order to reduce the conservatism of the asymptotic stability conditions. From the proof it can be seen that $\dot{V}(t)$ remains unaffected by the slack variables $T_{1}, T_{2}, Y_{1}, Y_{2}, Z_{1}, Z_{2}$, so these matrices provide a more flexible LMI condition in (11) and (15), reducing the conservatism of Theorem 1, and consequently that of Theorem 2. This advantage will be appreciated in the numerical examples at the end of this paper.

Remark 2. For given $\alpha$, the inequalities (11) and (15) are linear and the problem can easily be solved using the LMI-toolbox of MATLAB. To find an optimal value of $\alpha$, a numerical optimization algorithm can be employed to reduce the conservatism.

Remark 3. The conditions (14) and (17) are nonlinear, so they cannot be directly tested using the usual numerical tools (such as the LMI-toolbox of MATLAB). In Section 4 we show that it is it possible to transform the nonlinear conditions into matrix inequalities, introducing relaxation conditions.

\section{Optimization problems}

The proposed conditions in Theorems 1 and 2 are in LMI form, so that they can be easily considered in convex optimization problems. At the end of the paper, three problems of interest are solved to illustrate these results.

4.1. Maximization of disturbance tolerance. The objective is to maximize the $\mathcal{L}_{2}$-norm bound on the disturbance for which it can be ensured that the system trajectories remain bounded. Assuming that the initial condition is zero, this can be accomplished by the following convex optimization problem:

Minimize $\beta$ subject to (11)-(13), (15)-16). 
4.2. Maximization of disturbance attenuation. For a non-null positive bound on the $\mathcal{L}_{2}$-norm of the admissible disturbances (given by $\beta^{-1}=\omega^{-1}$ ), the objective is to minimize the upper bound of the $\mathcal{L}_{2}$-gain of $w(t)$ on $z(t)$. Assuming that the initial condition is zero, this can be obtained from the solution of the following convex optimization problem:

$$
\text { Minimize } \gamma \text { subject to (11)-13, (15)-16). }
$$

4.3. Maximization of the region of admissible initial conditions. Considering the disturbance-free case $(w(t)=0)$, this stage shows how the theoretical conditions derived so far can be cast as LMI-based optimization problems, to determine a suitable gain $K$ and the associated domain of attraction (so that the system trajectories starting from any initial functions $\phi(\theta)$ in $D_{e}$ will remain within $D_{e}$ for all $t>0$ ).

The idea is to develop an estimate of the largest possible domain of initial conditions satisfying $\varpi \leq$ $\beta^{-1}$ for which it can be ensured that the closed-loop system trajectories remain bounded. Then, we introduce new auxiliary matrix variables $\bar{\Pi}^{-1}=\widetilde{\Pi}$ with $\Pi=$ $P, Q_{1}, Q_{2}, R_{1}, R_{2}, W$ and $X^{-1}=\tilde{X}$, and impose the following conditions:

$$
\begin{aligned}
& {\left[\begin{array}{cc}
\sigma_{1} I & \widetilde{X} \\
\widetilde{X}^{T} & \widetilde{P}
\end{array}\right] \geq 0, \quad\left[\begin{array}{cc}
\sigma_{2} I & \widetilde{X} \\
\widetilde{X}^{T} & \widetilde{Q}_{1}
\end{array}\right] \geq 0,} \\
& {\left[\begin{array}{cc}
\sigma_{3} I & \widetilde{X} \\
\widetilde{X}^{T} & \widetilde{Q}_{2}
\end{array}\right] \geq 0, \quad\left[\begin{array}{cc}
\sigma_{4} I & \widetilde{X} \\
\widetilde{X}^{T} & \widetilde{R}_{1}
\end{array}\right] \geq 0,} \\
& {\left[\begin{array}{cc}
\sigma_{5} I & \widetilde{X} \\
\widetilde{X}^{T} & \widetilde{R}_{2}
\end{array}\right] \geq 0, \quad\left[\begin{array}{cc}
\sigma_{6} I & \widetilde{X} \\
\widetilde{X}^{T} & \widetilde{W}
\end{array}\right] \geq 0 .}
\end{aligned}
$$

Thus, the condition (14) implies that

$$
\begin{aligned}
\delta^{2}\left[\sigma_{1}+h_{m} \sigma_{2}+h_{k} \sigma_{3}\right. & +\frac{h_{m}^{2}}{2} \sigma_{4} \\
+ & \left.\frac{h_{k}^{2}}{2} \sigma_{5}+h_{m} \sigma_{6}\right] \leq \beta^{-1} .
\end{aligned}
$$

Therefore, a feasibility problem is constructed, based on the ideas presented by Lee et al. (2004), as follows:

$$
\begin{gathered}
\min \operatorname{tr}\left(\bar{P} \widetilde{P}+\bar{Q}_{1} \widetilde{Q}_{1}+\bar{Q}_{2} \widetilde{Q}_{2}+\bar{R}_{1} \widetilde{R}_{1}+\bar{R}_{2} \widetilde{R}_{2}\right. \\
\left.+\bar{W} \widetilde{W}+\left(X+X^{T}\right)\left(\widetilde{X}+\widetilde{X}^{T}\right)\right)
\end{gathered}
$$

subject to 111-13, (15)-16), 20, (21),

$$
\left[\begin{array}{cc}
\bar{\Pi} & * \\
I & \widetilde{\Pi}
\end{array}\right] \geq 0, \quad\left[\begin{array}{cc}
X+X^{T} & * \\
I & \tilde{X}+\tilde{X}^{T}
\end{array}\right] \geq 0 .
$$

The new LMI problem can be solved by using Algorithm 1.
Algorithm 1. Cone complementarity.

Step 1. Given $h_{m}$, choose a sufficiently small $\delta$ and set $\left(\bar{\Pi}, X, \widetilde{\Pi}, \widetilde{X}, \sigma_{1, \ldots, 6}\right)_{0}=\left(\bar{\Pi}, X, \widetilde{\Pi}, \widetilde{X}, \sigma_{1, \ldots, 6}\right)$ that satisfies the constrained minimization (22). Then, fix $\Delta$ where $\delta=\delta+\Delta$.

Step 2. Solve the following LMI minimization problem in the matrix variables $\bar{\Pi}$ and $\widetilde{\Pi}$ :

$$
\begin{aligned}
\min \operatorname{tr} & \bar{P} \widetilde{P}_{0}+\bar{Q}_{1} \widetilde{Q}_{1_{0}}+\bar{Q}_{2} \widetilde{Q}_{2_{0}}+\bar{R}_{1} \widetilde{R}_{1_{0}} \\
& +\bar{R}_{2} \widetilde{R}_{2_{0}}+\bar{W} \widetilde{W}_{0}+\left(X+X^{T}\right)\left(\widetilde{X}_{0}+\widetilde{X}_{0}^{T}\right) \\
& +\bar{P}_{0} \widetilde{P}+\bar{Q}_{1_{0}} \widetilde{Q}_{1}+\bar{Q}_{2_{0}} \widetilde{Q}_{2} \\
& +\bar{R}_{1_{0}} \widetilde{R}_{1}+\bar{R}_{2_{0}} \widetilde{R}_{2}+\bar{W}_{0} \widetilde{W} \\
& \left.+\left(X_{0}+X_{0}^{T}\right)\left(\widetilde{X}+\widetilde{X}^{T}\right)\right)
\end{aligned}
$$

subject to the LMIs in 22).

Step 3. Substitute the new matrix variables into (22). If the result is feasible, then set $\delta=\delta+\Delta$ and repeat Step 2; otherwise, $\delta=\delta-\Delta$ is the desired estimate: Stop.

\section{Illustrative examples}

This section illustrates the proposed methodology: the first two examples do not include uncertainty, in order to compare the results with those in the literature (Chen et al., 2015; Liu, 2011; Manitius, 1984), whereas the last one includes the uncertainty to show how the proposed solution can easily include uncertainty.

Example 1. (Chen et al., 2015) Consider a neutral time-delay system with an input limitation at \pm 15 , described as follows:

$$
\begin{aligned}
\dot{x}(t)-C \dot{x}(t-\tau(t))= & A x(t)+A_{d} x(t-\tau(t)) \\
& +B \operatorname{sat}(u(t)),
\end{aligned}
$$

where

$$
\begin{aligned}
& A=\left[\begin{array}{cc}
1 & 1.5 \\
0.3 & -2
\end{array}\right], \quad A_{d}=\left[\begin{array}{cc}
0 & -1 \\
0 & 0
\end{array}\right], \\
& B=\left[\begin{array}{c}
10 \\
1
\end{array}\right], \quad C=\left[\begin{array}{ll}
c & 0 \\
0 & c
\end{array}\right], \quad h_{m}=1 .
\end{aligned}
$$

Applying the stability results presented in Theorem 1 and the cone complementarity algorithm, taking $\beta=1$, $h_{k}=0.1$, the corresponding state-feedback gain obtained for the neutral system when $c=0.2$ and $d=0.1$ is given by $K=[-0.2492-0.0175]$. For a constant time-delay (i.e., when $c=d=0), K=\left[\begin{array}{ll}-0.2426 & -0.0829\end{array}\right]$ is obtained: for these calculations the tuning parameters were fixed to be $\alpha=0.3$ and $\alpha=0.5$, respectively.

Table 11 provides a comparison with previous approaches: it is clear that the obtained stability radius $\delta$ is significantly larger than those obtained by Da Silva 
et al. (2011), Chen et al. (2015), El Haoussi et al. (2013) or Dey et al. (2014). To see the effect of varying $\alpha$, the gains are listed in Table 2 for the neutral system.

Table 1. Comparison of stability radius $\delta$ for Example 1 .

\begin{tabular}{|c|c|c|}
\hline Approach & $\delta(c=0.2)$ & $\delta(c=0)$ \\
\hline \hline Da Silva et al., 2011 & 70.74 & 83.55 \\
Chen et al., 2015 & 76.23 & 84.61 \\
El Haoussi et al., 2013 & 80.21 & 96.16 \\
Dey et al., 2014 & - & 106.29 \\
Theorem11 & 97.0 & 115.0 \\
\hline
\end{tabular}

Table 2. Gain obtained for different values of $\alpha$ for Example 1.

\begin{tabular}{|c|c|}
\hline Tuning parameter & $K(c=0.2, \delta=97)$ \\
\hline \hline$\alpha=0.1$ & Infeasible \\
$\alpha=0.2$ & {$[-0.2557-0.0472]$} \\
$\alpha=0.3$ & {$[-0.2492-0.0175]$} \\
$\alpha=0.4$ & Infeasible \\
$\vdots$ & $\vdots$ \\
$\alpha=0.9$ & Infeasible \\
\hline
\end{tabular}

To see graphically the improvements of the proposed approach, Fig. 1 presents the domains of attraction of the controllers obtained with the different approaches (Table 1) for both the neutral system $(c=0.2)$ and the time-delay system $(c=0)$.

Example 2. To show the applicability of the technique to realistic problems, we now consider the control of the Mach number in a wind tunnel. An approximated nominal model of this system is the following (which takes into account practical limitations in the range of the guide vane angle actuator (El Fezazi et al., 2017; Manitius, 1984)):

$$
\begin{aligned}
\dot{x}(t)= & {\left[\begin{array}{ccc}
-a & 0 & 0 \\
0 & 0 & 1 \\
0 & -w^{2} & -2 \xi w
\end{array}\right] x(t) } \\
& +\left[\begin{array}{ccc}
0 & k a & 0 \\
0 & 0 & 0 \\
0 & 0 & 0
\end{array}\right] x(t-\tau(t)) \\
& +\left[\begin{array}{c}
0 \\
0 \\
w^{2}
\end{array}\right] \operatorname{sat}(u(t)) .
\end{aligned}
$$

To draw a comparison with previous results, the numerical matrices given by Manitius (1984) and Liu (2011) are used, with $u_{0}=1, d=0$ and $h_{k}=1$. Applying Theorem 1 gives the following gain matrix, when $\alpha=0.7$ and $\beta=1$ :

$$
K=\left[\begin{array}{lll}
0.0442 & 0.0866 & 0.0122
\end{array}\right]
$$

which stabilizes the system for any delay smaller than $h_{m}=3$; the stability radius for the set of acceptable initial conditions is $\delta=6$. For comparison with the results of Manitius (1984) and Liu (2011), the admissible upper bounds of the delay are summarized in Table 3 , the proposed method produces controllers that ensure stability for a significantly larger delay than those previously published in the literature.

Example 3. We retake the Mach number control problem presented in Example 2: as in reality the linear model used in Example 2 is just an approximation, we now consider uncertainties and disturbances in the model, to make the design more realistic. That is, the following uncertain model is now used:

$$
\begin{aligned}
\dot{x}(t)= & \widehat{A} x(t)+\widehat{A}_{d} x(t-\tau(t))+\widehat{B} \mathrm{sat}(u(t)) \\
& +B_{w} w(t) \\
z(t)= & C_{z} x(t)
\end{aligned}
$$

with

$$
\begin{gathered}
B_{w}=\left[\begin{array}{c}
0 \\
0 \\
10
\end{array}\right], \quad C_{z}=\left[\begin{array}{lll}
1 & 0 & 0 \\
0 & 1 & 0
\end{array}\right], \\
F=\sin (t), \quad D=I, \\
E_{0}=0.02 A, \quad E_{1}=0.02 A_{d}, \quad E_{2}=0.02 B .
\end{gathered}
$$

Taking $u_{0}=1, d=0, h_{k}=1, \alpha=0.1$, and $\varepsilon=$ 0.1 , the algorithm proposed in (18), based on Theorem 2 , successfully finds the following feedback stabilizing gain:

$$
K=\left[\begin{array}{lll}
-0.0008 & 2.6169 & 0.4760
\end{array}\right] \times 10^{-3},
$$

with the optimal value and the upper bound to the delay being $\beta=10^{-4}$ and $h_{m}=8$, respectively.

Applying the algorithm proposed in 19 and based on Theorem 2], the prescribed scalar is $\gamma=0.2$ where $u_{0}=1, d=0, h_{k}=1, \beta=1, \alpha=0.1$, and $\varepsilon=0.1$. For $h_{m}=5$, the feedback stabilizing gain is given by

$$
K=\left[\begin{array}{lll}
-10.0289 & 4.5958 & 0.2255
\end{array}\right] \times 10^{-3} .
$$

Some simulation results are now presented in Figs. 2-3 for this example, with $x(0)=\left[\begin{array}{lll}-5 & 5 & -5\end{array}\right]^{T}$ and the following disturbance added to the system:

$$
w(t)= \begin{cases}10, & 0 \leq t \leq 1 \\ 0, & t \geq 1\end{cases}
$$

Table 3. Comparison of maximum allowable delay $h_{m}$ for Example 2.

\begin{tabular}{|c|c|c|c|}
\hline Method & Manitius, 1984 & Liu, 2011 & This paper \\
\hline \hline$h_{m}$ & 0.33 & 0.97 & 3.00 \\
\hline
\end{tabular}




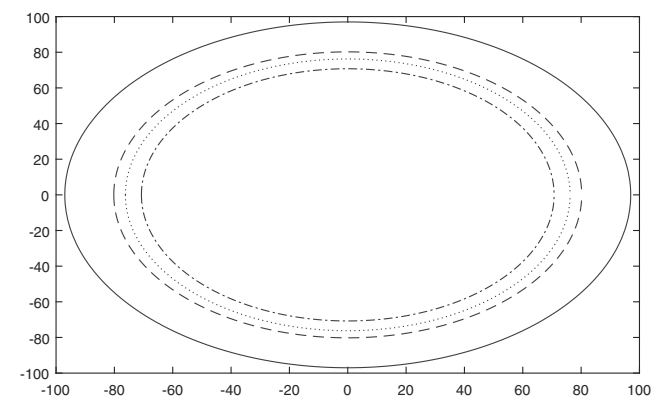

(a)

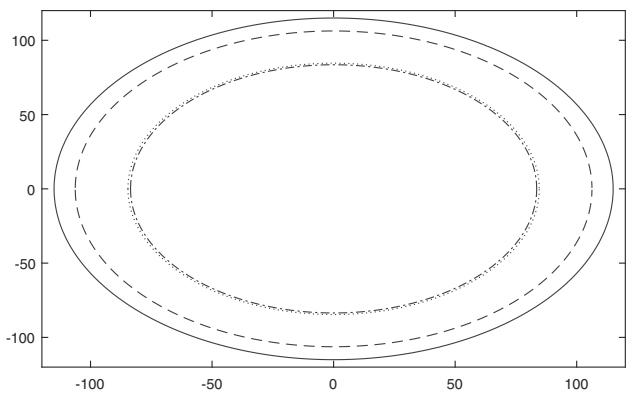

(b)

Fig. 1. Comparison of the domains of attraction obtained with the methods presented in Table 1 for the neutral system (a) and the time-delay system (b).

It can be seen that the controller performs adequately: even though the control input is initially saturated, the states are driven to the origin and the disturbance is correctly compensated. In fact, very good transient responses are obtained.

\section{Conclusions}

A methodology for robust stabilization of uncertain neutral time-delay systems with saturating inputs has been presented, where the controller uses sampled measurements. Conditions are provided as LMIs, which depend on the largest expected delay, that guarantee the stability of the closed-loop system when the initial states are taken within a region of attraction that is simultaneously estimated. The proposed conditions have been illustrated by numerical examples, which showed the feasibility of the proposed approach, and the fact that it is less conservative than those previously proposed in the literature. The feedback design method presented here has a wide range of applicability, as the class of systems investigated appears in many process control applications: this has been illustrated by application to the feedback control of the Mach number in a wind tunnel.

The proposed approach open new lines of research: in the near future, we aim to extend the proposed methodology to related problems such as anti-windup synthesis for uncertain systems, observer-based controller design for uncertain neutral systems, limitations in the increment or rate of control signals, etc.

\section{Acknowledgment}

This work was partly funded by the MiCInn project DPI2014-54530-R.

\section{References}

Cao, Y.-Y., Lin, Z. and Hu, T. (2002). Stability analysis of linear time-delay systems subject to input saturation, IEEE Transactions on Circuits and Systems 49(2): 233-240.

Chen, Y., Fei, S. and Li, Y. (2015). Stabilization of neutral time-delay systems with actuator saturation via auxiliary time-delay feedback, Automatica 52: 242-247.

Da Silva, J.M.G., Seuret, A., Fridman, E. and Richard, J.P. (2011). Stabilization of neutral systems with saturating control inputs, International Journal of Systems Science 42(7): 1093-1103.

Dey, R., Ghosh, S., Ray, G. and Rakshit, A. (2014). Improved delay-dependent stabilization of time-delay systems with actuator saturation, International Journal of Robust and Nonlinear Control 24(5): 902-917.

El Fezazi, N., El Haoussi, F., Tissir, E.H., Alvarez, T. and Tadeo, F. (2017). Robust stabilization using LMI techniques of neutral time-delay systems subject to input saturation, Journal of Physics: Conference Series 783(1): 012031.

El Fezazi, N., Lamrabet, O., El Haoussi, F., Tissir, E.H., Alvarez, T. and Tadeo, F. (2016). Robust controller design for congestion control in TCP/IP routers, 5th IEEE International Conference on Systems and Control, Marrakesh, Morocco, pp. 243-249.

El Fezazi, N., El Haoussi, F., Tissir, E.H., Husain, A.R. and Zakaria, M.I. (2016). Delay-dependent robust anti-windup synthesis approach to AQM in TCP/IP networks, 2nd IEEE International Conference on Electrical and Information Technologies, Tanger, Morocco, pp. 294-299.

El Fezazi, N., El Haoussi, F., Tissir, E. H. and Tadeo, F. (2015). Delay dependent anti-windup synthesis for time-varying delay systems with saturating actuators, International Journal of Computer Applications 111(1): 1-6.

El Haoussi, F., Tissir, E.H., Tadeo, F. and Hmamed, A. (2013). Robust stabilization with saturating actuators of neutral and state delayed systems, International Journal of Sciences and Techniques of Automatic Control and Computer Engineering 7(1): 1878-1889. 


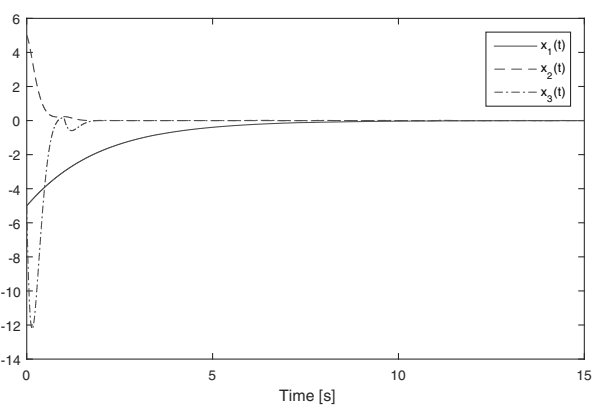

(a)

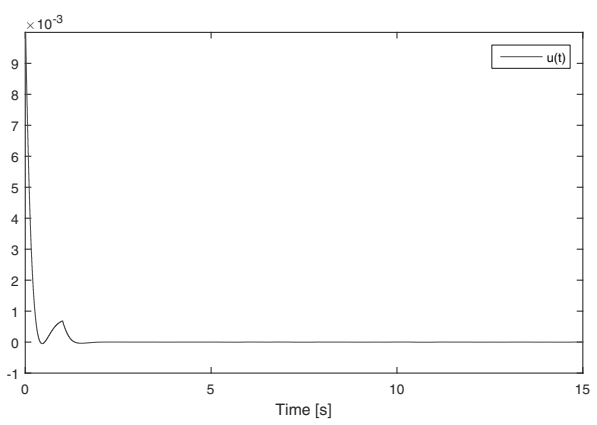

(b)

Fig. 2. Evolution of the state variables (a) and the input (b) for Example 3 (based on (18)).

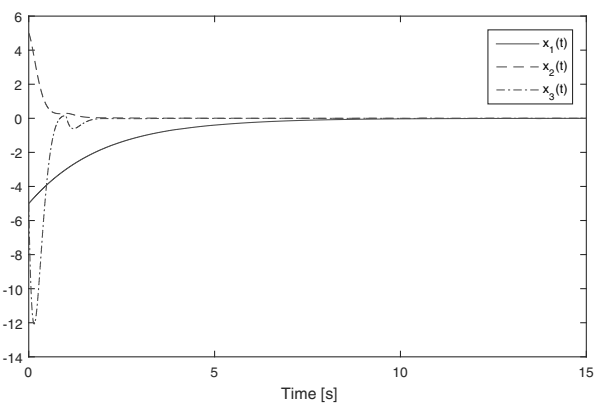

(a)

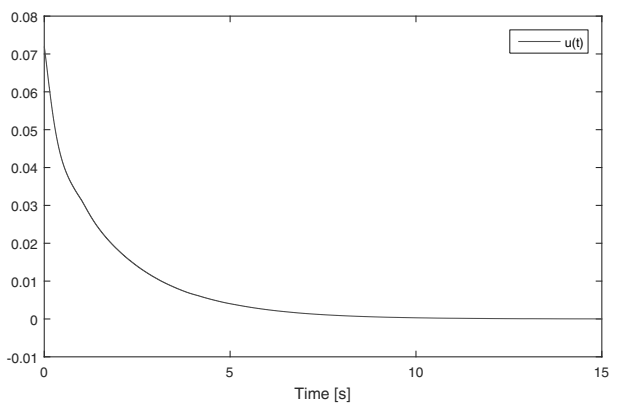

(b)

Fig. 3. Evolution of the state variables (a) and the input (b) for Example 3 (based on (19)).

El Haoussi, F. and Tissir, E.H. (2010). Delay and its time-derivative dependent robust stability of uncertain neutral systems with saturating actuators, International Journal of Automation and Computing 7(4): 455-462.

Fridman, E., Seuret, A. and Richard, J.P. (2004). Robust sampled-data stabilization of linear systems: An input delay approach, Automatica 40(8): 1441-1446.

Gu, K., Chen, J. and Kharitonov, V. (2003). Stability of TimeDelay Systems, Springer Science and Business Media, Basel.

Lee, Y.S., Moon, Y.S., Kwon, W.H. and Park, P.G. (2004). Delay-dependent robust $\mathrm{H}_{\infty}$ control for uncertain systems with a state-delay, Automatica 40(1): 65-72.

Liu, P.L. (2011). Delay-dependent stabilization for linear time-delay uncertain systems with saturating actuators, International Journal of General Systems 40(3): 301-312.

Liu, P.-L. (2005). Delay-dependent asymptotic stabilization for uncertain time-delay systems with saturating actuators, International Journal of Applied Mathematics and Computer Science 15(1): 45-51.

Manitius, A.Z. (1984). Feedback controllers for a wind tunnel model involving a delay: Analytical design and numerical simulation, IEEE Transactions on Automatic Control 29(12): 1058-1068.

Mesquine, F., Tadeo, F., and Benzaouia, A. (2004). Regulator problem for linear systems with constraints on control and its increment or rate, Automatica 40(8): 1387-1395.
Peterson, I.R. (1987). A stabilization algorithm for a class of uncertain linear systems, Systems and Control Letters 8(4): 351-357.

Seuret, A. and Da Silva, J.M.G. (2012). Taking into account period variations and actuator saturation in sampled-data systems, Systems and Control Letters 61(12): 1286-1293.

Tarbouriech, S., Da Silva, J.M.G. and Garcia, G. (2003). Delay-dependent anti-windup loops for enlarging the stability region of time-delay systems with saturating inputs, Journal of Dynamic Systems, Measurement, and Control 125(2): 265-267.

Thuan, M.V., Phat, V.N. and Trinh, H. (2012). Observer-based controller design of time-delay systems with an interval time-varying delay, International Journal of Applied Mathematics and Computer Science 22(4): 921-927, DOI: 10.2478/v10006-012-0068-8.

Tissir, E.H. (2010). Delay-dependent robust stabilization for systems with uncertain time varying delay, Journal of Dynamic Systems, Measurement, and Control 132(5): 054504.

Tissir, E.H. and Hmamed, A. (1992). Further results on the stabilization of time delay systems containing saturating actuators, International Journal of Systems Science 23(4): 615-622.

Wang, H.P., Tian, Y. and Christov, N. (2014). Piecewise-continuous observers for linear systems with sampled and delayed output, International Journal of Systems Science 47(8): 1804-1815. 
Wang, H.P., Tian, Y. and Vasseur, C. (2015). Piecewise continuous hybrid systems based observer design for linear systems with variable sampling periods and delay output, Signal Processing 114: 75-84.

Zakaria, M.I., Husain, A.R., Mohamed, Z., El Fezazi, N. and Shah, M.B.N. (2015). Lyapunov-Krasovskii stability condition for system with bounded delay-an application to steer-by-wire system, 5th IEEE International Conference on Control System, Computing and Engineering, Penang, Malaysia, pp. 543-547.

Zhang, X.-M., Wu, M., She, J.-H. and He, Y. (2005). Delay-dependent stabilization of linear systems with time-varying state and input delays, Automatica 41(8): 1405-1412.

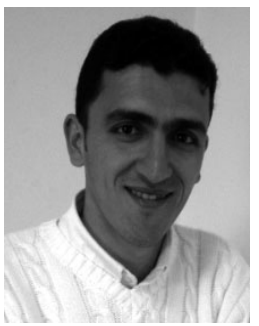

Nabil El Fezazi received his Master's degree in automated industrial systems engineering from Sidi Mohammed Ben Abdellah University, Faculty of Sciences, Morocco, in 2013. He is currently a $\mathrm{PhD}$ student at the same faculty. His main research interests are robust and $\mathrm{H}_{\infty}$ control, sampled-data control, steer-by-wire systems, TCP/IP networks and wind tunnels.

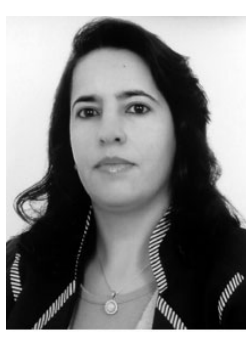

Fatima El Haoussi received her $\mathrm{PhD}$ degree from Sidi Mohammed Ben Abdellah, University Faculty of Sciences, Morocco, in 2008. She is now a professor at the Polydisciplinary Faculty of Nador, Morocco. Her research interests include robust and $\mathrm{H}_{\infty}$ control, sampled-data control, teleoperation systems, systems with saturating actuators, time delay systems, TCP/IP networks, wind tunnels.

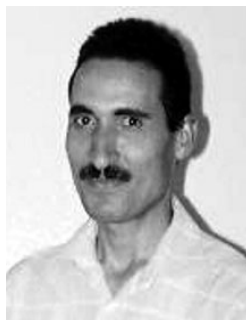

El Houssaine Tissir received the diploma of higher studies and the doctorate of state degree from Sidi Mohammed Ben Abellah University, Faculty of Sciences, Morocco, in 1992 and 1997, respectively. He is now a professor at Sidi Mohammed Ben Abdellah University. His research interests include robust and $\mathrm{H}_{\infty}$ control, singular systems, switched systems, time delay systems, systems with saturating actuators and TCP/IP networks.

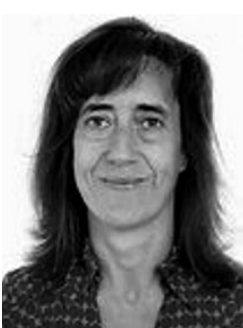

Teresa Alvarez is an associate professor at the School of Engineering of the University of Valladolid, Spain (since 1995). She graduated from the University of Oviedo in computer science in 1989, from the University of Valladolid in computer engineering in 1992, and from the University of Bradford, UK, in control engineering (MSc). She obtained her PhD in automatic control from the University of Valladolid in 1994. Her current interest lies in computer-controlled systems, focusing on congestion control in computer networks.

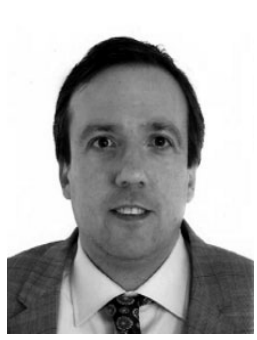

Fernando Tadeo has been a professor at the School of Engineering, University of Valladolid, Spain, since 2010. He graduated from the same university in physics and in electronic engineering. After completing his MSc in control engineering at the University of Bradford, UK, he obtained his $\mathrm{PhD}$ from the University of Valladolid. His main interest area is advanced process control, focusing on applications in desalination and renewable energies (wind, solar and osmotic).

\section{Appendix A}

Computing the time derivative of the functional (9) along the trajectory of the system $(7)$ gives

$$
\begin{aligned}
J(t) \leq & 2 x^{T}(t) P \dot{x}(t)+x^{T}(t)\left(Q_{1}+Q_{2}\right) x(t) \\
& +\dot{x}^{T}(t)\left(h_{m} R_{1}+h_{k} R_{2}+W\right) \dot{x}(t) \\
& -(1-d) x^{T}(t-\tau(t)) Q_{1} x(t-\tau(t)) \\
& -(1-d) \dot{x}^{T}(t-\tau(t)) W \dot{x}(t-\tau(t)) \\
& -\int_{t-\tau(t)}^{t} \dot{x}^{T}(s) R_{1} \dot{x}(s) \mathrm{d} s+\frac{1}{\gamma} z^{T}(t) z(t) \\
& -\int_{t-d_{k}(t)}^{t} \dot{x}^{T}(s) R_{2} \dot{x}(s) \mathrm{d} s-w^{T}(t) w(t) .
\end{aligned}
$$

Using the free weighting matrix approach, for appropriately dimensioned matrices $T_{1}$ and $T_{2}$, we have

$$
\begin{gathered}
2\left[x^{T}(t) T_{1}+\dot{x}^{T}(t) T_{2}\right][-\dot{x}(t)+C \dot{x}(t-\tau(t)) \\
+A x(t)+A_{d} x(t-\tau(t))+B K x\left(t-d_{k}(t)\right) \\
\left.-B \psi\left(K x\left(t-d_{k}(t)\right)\right)+B_{w} w(t)\right]=0 .
\end{gathered}
$$

Then, applying Lemmas 2 and 3 , and taking account of (A1), we get

$$
J(t) \leq \eta^{T}(t) \Psi \eta(t)
$$

where

$$
\Psi=\left[\begin{array}{ccccccc}
\Psi_{11} & * & * & * & * & * & * \\
\Psi_{21} & \Psi_{22} & * & * & * & * & * \\
\Psi_{31} & \Psi_{32} & \Psi_{33} & * & * & * & * \\
\Psi_{41} & \Psi_{42} & 0 & \Psi_{44} & * & * & * \\
\Psi_{51} & \Psi_{52} & 0 & 0 & \Psi_{55} & * & * \\
\Psi_{61} & \Psi_{62} & 0 & \Psi_{64} & 0 & \Psi_{66} & * \\
\Psi_{71} & \Psi_{72} & 0 & 0 & 0 & 0 & \Psi_{77}
\end{array}\right]
$$

$$
\begin{aligned}
\eta(t)=\left[\begin{array}{llll}
x^{T}(t) & \dot{x}^{T}(t) & x^{T}(t-\tau(t)) & x^{T}\left(t-d_{k}(t)\right) \\
& \dot{x}^{T}(t-\tau(t)) & \psi^{T}\left(K x\left(t-d_{k}(t)\right)\right) & w^{T}(t)
\end{array}\right]^{T},
\end{aligned}
$$


and

$$
\begin{aligned}
\Psi_{11}= & T_{1} A+A^{T} T_{1}^{T}+Y_{1}+Y_{1}^{T} \\
& +Z_{1}+Z_{1}^{T}+Q_{1}+Q_{2}, \\
& +h_{m} Y_{1} R_{1}^{-1} Y_{1}^{T}+h_{k} Z_{1} R_{2}^{-1} Z_{1}^{T}, \\
& +\frac{1}{\gamma} C_{z}^{T} C_{z}, \\
\Psi_{21}= & -T_{1}^{T}+T_{2} A+P, \\
\Psi_{32}= & A_{d}^{T} T_{2}^{T}, \\
\Psi_{77}= & -I \\
\Psi_{22}= & -T_{2}-T_{2}^{T}+h_{m} R_{1}+h_{k} R_{2}+W, \\
\Psi_{31}= & A_{d}^{T} T_{1}^{T}-Y_{1}^{T}+Y_{2}+h_{m} Y_{2} R_{1}^{-1} Y_{1}^{T}, \\
\Psi_{33}= & -Y_{2}-Y_{2}^{T}-(1-d) Q_{1}+h_{m} Y_{2} R_{1}^{-1} Y_{2}^{T}, \\
\Psi_{41}= & K^{T} B^{T} T_{1}^{T}-Z_{1}^{T}+Z_{2}+h_{k} Z_{2} R_{2}^{-1} Z_{1}^{T}, \\
\Psi_{42}= & K^{T} B^{T} T_{2}^{T}, \\
\Psi_{44}= & -Z_{2}-Z_{2}^{T}+h_{k} Z_{2} R_{2}^{-1} Z_{2}^{T}, \\
\Psi_{51}= & C^{T} T_{1}^{T}, \\
\Psi_{52}= & C^{T} T_{2}^{T}, \\
\Psi_{55}= & -(1-d) W, \\
\Psi_{61}= & -B^{T} T_{1}^{T}, \\
\Psi_{62}= & -B^{T} T_{2}^{T}, \\
\Psi_{64}= & T_{0} G, \\
\Psi_{66}= & -2 T_{0}, \\
\Psi_{71}= & B_{w}^{T} T_{1}^{T}, \\
\Psi_{72}= & B_{w}^{T} T_{2}^{T} . \\
& -1, \\
&
\end{aligned}
$$

It is clear that, if $\Psi<0$, then

$$
J(t)=\dot{V}(t)+\frac{1}{\gamma} z^{T}(t) z(t)-w^{T}(t) w(t)<0 .
$$

From $\Psi$, it is easy to see that $T_{2}$ is nonsingular and consequently $T_{1}$ is inversible. Then, setting $T_{2}=\alpha T_{1}$, applying the Schur complement to A2, premultiplying it by $\operatorname{diag}\left\{T_{1}^{-1}, T_{1}^{-1}, T_{1}^{-1}, T_{1}^{-1}, T_{1}^{-1}, T_{0}^{-1}, I, T_{1}^{-1}, T_{1}^{-1}, I\right\}$ and postmultiplying by its transpose, and then introducing the change of variables $X=T_{1}^{-1}, U=K X^{T}$, $M=G X^{T}, S=T_{0}^{-1}$, and $\bar{\Pi}=X \Pi X^{T}$, where $\Pi=P, Q_{1}, Q_{2}, R_{1}, R_{2}, W, Y_{1}, Y_{2}, Z_{1}, Z_{2}$, give (111).

By virtue of 111, when $w(t)=0$, A3 implies that $\dot{V}(t)<0$, so it follows that the system (7) is asymptotically stable. Now, when $w(t) \neq 0$, integrating both the sides of (A3) from 0 to $T$, we have

$$
V(T)-V(0)+\int_{0}^{T}\left(\frac{1}{\gamma} z^{T}(t) z(t)-w^{T}(t) w(t)\right] \mathrm{d} t<0 .
$$

Let $T \rightarrow \infty$. Since the system is asymptotically stable we get $V(T)=0$, and using the zero initial condition, we can conclude that (8) is satisfied.

On the other hand, the satisfaction of (12) guarantees that $\forall x(t) \in D_{e}, x(t) \in \mathcal{S}$. In fact, $D_{e} \subset \mathcal{S}$ is met by the following conditions:

$$
\left[\begin{array}{cc}
P & * \\
K_{i}-G_{i} & \beta u_{0_{i}}^{2}
\end{array}\right] \geq 0 .
$$

Pre- and postmultiplying (A4) respectively by $\Delta=$ $\operatorname{diag}\{X, I\}$ and its transpose will result in the LMI (12).

Moreover, the satisfaction of (13) and (14) can be proven as follows: from the $\mathrm{L}-\mathrm{K}$ functional defined in (9), we have

$$
\begin{aligned}
V(0) \leq & x^{T}(0) P x(0)+\int_{-h_{m}}^{0} x^{T}(s) Q_{1} x(s) \mathrm{d} s \\
& +\int_{-h_{k}}^{0} x^{T}(s) Q_{2} x(s) \mathrm{d} s \\
& +\int_{-h_{m}}^{0} \int_{\theta}^{0} \dot{x}^{T}(s) R_{1} \dot{x}(s) \mathrm{d} s \mathrm{~d} \theta \\
& +\int_{-h_{k}}^{0} \int_{\theta}^{0} \dot{x}^{T}(s) R_{2} \dot{x}(s) \mathrm{d} s \mathrm{~d} \theta \\
& +\int_{-h_{m}}^{0} \dot{x}^{T}(s) W \dot{x}(s) \mathrm{d} s .
\end{aligned}
$$

Then we obtain

$V(0)$

$$
\begin{aligned}
\leq & \left(\bar{\lambda}(P)+h_{m} \bar{\lambda}\left(Q_{1}\right)+h_{k} \bar{\lambda}\left(Q_{2}\right)\right]\|\phi(\theta)\|^{2} \\
& +\left(\frac{h_{m}^{2}}{2} \bar{\lambda}\left(R_{1}\right)+\frac{h_{k}^{2}}{2} \bar{\lambda}\left(R_{2}\right)+h_{m} \bar{\lambda}(W)\right]\|\dot{\phi}(\theta)\|^{2}
\end{aligned}
$$$$
=\varpi \text {. }
$$

Therefore, we have $x^{T}(t) P x(t) \leq V(t) \leq V(0)+$ $\|w(t)\|_{2}^{2} \leq \varpi+\omega^{-1} \leq \beta^{-1}$; that is, for all $t \geq 0$, the trajectories of the system do not leave the set $D_{e}$ for any initial functions $\phi(\theta)$ in $D_{e}$, which ensures that $x(t) \in \mathcal{S}$.

\section{Appendix B}

Replace $A, A_{d}$, and $B$ by $A+D F(t) E_{0}, A_{d}+D F(t) E_{1}$, and $B+D F(t) E_{2}$, respectively. We find that Eqn. (11) is equivalent to $\Omega+2 \Upsilon F(t) \Phi<0$, where

$$
\begin{aligned}
& \Upsilon=\left[\begin{array}{cccccccccc}
D^{T} & \alpha D^{T} & 0 & 0 & 0 & 0 & 0 & 0 & 0 & 0
\end{array}\right]^{T}, \\
& \Phi=\left[\begin{array}{llllllll}
E_{0} X^{T} & 0 & E_{1} X^{T} & E_{2} U & 0 \\
& 8
\end{array}\right.
\end{aligned}
$$


According to Lemma 1 and by the Schur complement, we obtain the LMI (15). This completes the proof.

Received: 26 January 2017

Revised: 11 May 2017

Accepted: 9 August 2017 